Delinda E. Mercer George Woody

\title{
Psychotherapie bei Kokainabhängigkeit
}

\section{Psychotherapy for Cocaine Dependence}

\section{Zusammenfassung}

Psychotherapeutische Ansätze in der Suchtbehandlung sind weit verbreitet, aber bisher wenig wissenschaftlich evaluiert worden. Es werden zunächst therapeutische Settings und Ansätze als auch Qualitäten der Therapeuten beschrieben, die sich in der Psychotherapie bewährt haben. Anschließend werden drei verhaltenstherapeutische Modelle für Kokainabhängigkeit dargestellt: individuelle Drogenberatung, kognitive Verhaltenstherapie, gemeindenahes Verstärkermodell. Zuletzt werden die vier Interventionen der NIDA-Kokain-Therapiestudie evaluiert, die alle zu einer Reduzierung des Konsums und Verbesserungen in anderen Bereichen führten.

\section{Schliuisselwörter}

Psychotherapie · Kognitive Verhaltenstherapie · Kokainabhängigkeit

\section{Abstract}

Psychotherapeutic interventions are frequently used in addiction treatment, however they have been seldomly evaluated. This paper presents therapeutic settings and techniques as well as qualities of therapists, that have shown to be effective. It further describes three behavioral models for cocaine dependence: individual drug counseling, cognitive-behavioral therapy, community reinforcement approach. Then the four interventions in the NIDA-cocaine-treatment study are evaluated, which all led to a reduction of cocaine use and improvements in other areas.

Key words

Psychotherapy · Cognitive Behavioral Therapy · Cocaine Dependence

\section{Einführung}

Seit dem Gipfel 1985 mit 5,7 Millionen Kokainkonsumenten ist die Anzahl in den USA zwar stark zurückgegangen, jedoch ist die Rate der regelmäßigen Konsumenten in der Bevölkerung seit 1992 stetig bei 1,5 Millionen bzw. 0,7\% der Bevölkerung geblieben [1]. Dies zeigt, dass Kokainabhängigkeit in den USA als Problem weiterhin besteht und sich auf gleichem Niveau hält. Gleichfalls wird deutlich, dass Kokainabhängigkeit offenbar schwierig zu behandeln ist. Mit zahlreichen Studien fördert das United States National Institute on Drug Abuse seit einigen Jahren die Erforschung der Behandlung von Kokainabhängigkeit unter Einschluss der neuesten Entwicklungen auf dem Gebiet der Pharmakologie sowie der Verhaltens- und psychosozialen Therapien.

Fast alle Behandlungsprogramme substanzbedingter Erkrankungen beinhalten auch eine psychosoziale Behandlung. Dabei ist Drogenberatung in einem gruppentherapeutischen Setting wahrscheinlich am weitesten verbreitet, ebenso wie individuelle Beratung oder Psychotherapie und Familientherapie in vielen drogenfreien Rehabilitations-, Methadonsubstitutions- und Entgiftungsprogrammen verfügbar sind [2]. Die Art der Psychotherapie variiert zwar, häufig angeboten werden jedoch kognitive Verhaltenstherapie, psychodynamische Kurztherapie und interpersonelle Therapie. 
Psychotherapeutische Ansätze zur Behandlung von Substanzabhängigkeit sind somit weit verbreitet, jedoch sind diese Ansätze erst in den letzten beiden Jahrzehnten wissenschaftlich evaluiert worden. Frühere Studien zur Suchttherapie konzentrierten sich meist auf die pharmakologische Behandlung, obwohl fast jedes Behandlungsprogramm psychosoziale Interventionen in irgendeiner Form zumindest mit einschloss und sie in manchen Fällen sogar das gesamte Programm ausmachten [3]. Diese relative Vernachlässigung der Erforschung und Evaluierung psychosozialer Behandlungen bei Substanzabhängigkeit beruhte zumindest teilweise auch auf den Schwierigkeiten, Forschung in diesem Bereich durchzuführen, z.B. verursacht durch Compliance-Probleme in Bezug auf die Studiendurchführung sowie Probleme bei der Rekrutierung und Haltequote der Patienten.

Psychotherapie wird in der einen oder anderen Form entweder allein oder in Verbindung mit Medikamenten bei der Behandlung psychiatrischer Störungen eingesetzt. Man sollte jedoch annehmen, dass die Psychotherapie einen Platz in der Suchtbehandlung haben müsste, da subjektive Befindlichkeitsstörungen sowohl als Mitverursacher als auch als Folge von Suchtstörungen gelten. Klinische Beobachtungen zeigen, dass einige Suchtmittel, zumindest zeitweilig, psychiatrische Symptome reduzieren und daher ihren Konsum fördern. Dieser Gebrauch zur Erleichterung von emotionalem Leiden resultierte in einer Theorie süchtigen Verhaltens, der so genannten „SelbstmedikationsHypothese“ [4].

Psychotherapie hat vielleicht die besten Erfolgschancen, wenn sie in ein laufendes Programm integriert wird, das direkt auf die Reduktion oder Eliminierung des Drogengebrauchs und der psychiatrischen Symptome, die die Abhängigkeit begleiten, abzielt.

\section{Therapeutische Settings}

Es haben sich zwei verschiedene Settings ergeben, die bei der Durchführung einer Psychotherapie - unter Berücksichtigung der notwendigen Drogenberatung - sinnvoll erscheinen. Washton [5] hat die Komponenten eines strukturierten, progressiven Behandlungsprogramms beschrieben: Es ist abstinenzorientiert, informiert über die Auswirkung von Drogen, fördert die Einbeziehung der Familie, bietet Gruppen- und individuelle Therapie an, unterstützt die Teilnahme an 12-Schritte-Programmen, fördert die körperliche Gesundheit und gleichfalls werden häufige Urinkontrollen durchgeführt. In solchen Programmen erfolgen Drogenberatung und Psychotherapie durch nur eine Person.

In einem anderen Behandlungsmodell werden dem Patienten ein Drogenberater und ein Psychotherapeut gesondert zugeteilt. Der Drogenberater kümmert sich um die konkreteren Bedürfnisse des Patienten, beispielsweise durch Gespräche über aktuelle Probleme, Unterstützung und Ermutigung der Bemühungen, den Drogenkonsum zu reduzieren, Überwachung/Registrierung der Fortschritte, Kontaktaufnahme oder Beratung mit medizinischem Personal, Hilfe bei der Arbeitssuche, Beschaffung eines Rechtsbeistandes, Unterstützung von Zielsetzungen durch Durchsetzung der Programmregeln und eine genaue Dokumentation. Wichtig für dieses Modell sind die Koordination der Angebote und gute persönliche Beziehungen zwischen Psychotherapeuten und Beratungspersonal.

\section{Therapeutische Qualitäten}

Die Qualitäten des Therapeuten haben einen wichtigen Einfluss auf den Erfolg der Behandlung [6]. Kleinman et al. [7] konnten zeigen, dass die Zuordnung des Therapeuten der stärkste Prädiktor für die Haltequote in der Behandlung von Kokainabhängigen war.

Insbesondere für drei Qualitäten des Therapeuten konnte ein Einfluss festgestellt werden:

- die Einstellung des Therapeuten,

- die Fähigkeiten des Therapeuten,

- das Interesse des Therapeuten, den Patienten helfen zu wollen [8].

All diese Qualitäten beeinflussen und formieren auch die Therapeuten-Patienten-Beziehung. Verschiedene Studien haben gezeigt, dass Therapeuten, die vom Beginn der Behandlung an eine Beziehung herstellten, die vom Patienten als „Helfen“ wahrgenommen wurde, eine bessere Chance auf Erfolg hatten als Therapeuten, die weniger positive Verbindungen herstellten [8].

Im Vordergrund dabei stehen das Interesse und der Umgang des Therapeuten mit bestimmten Problemen. Manche Therapeuten zeigen stark negative Reaktionen auf manipulierendes, antisoziales, impulsives und forderndes Verhalten, welches drogenabhängige Patienten zeigen können. Therapeuten mit überwiegend negativen Reaktionen werden diese Patienten letztlich nicht erfolgreich behandeln können.

\section{Therapeutische Ansätze}

Die meisten Techniken individueller Psychotherapie für drogenabhängige Patienten basieren auf Modellen aus der Behandlung von psychiatrischen Störungen, insbesondere der Depression. Für eine effektive Therapie müssen Suchttherapien jedoch einige konkretere Verhaltensregeln, die normalerweise als Teil der Suchtberatung angesehen werden, integrieren.

Zwar unterscheidet sich Kokainabhängigkeit von anderen Substanzabhängigkeiten, dennoch sind viele dieser Komponenten oder Interventionen auch bei Abhängigkeit von anderen Substanzen geeignet. Dies gilt ebenso für die meisten Verhaltenstherapien, die, häufig nur mit geringfügigen Änderungen, auf alle Substanzabhängigkeiten anwendbar sind.

Zusammenfassend sollte eine effektive Therapie von Kokainabhängigkeit Folgendes beinhalten. Sie sollte:

1. zugeschnitten sein auf individuelle Probleme und Bedürfnisse

2. einfach und schnell erreichbar sein/niedrigschwellig

3. sich sowohl mit den Problemen befassen, die mit dem Drogenkonsum verbunden sind (medizinisch, psychologisch, sozial, Arbeit, familiär, rechtlich) wie mit dem Drogenkonsum selbst

4. sich den sich ändernden Behandlungsbedürfnissen des Klienten anpassen

5. eine ausreichende Behandlungsdauer haben (3 Monate und mehr)

6. Beratung oder andere psychosoziale Komponenten einbeziehen 
7. wenn möglich, eine kombinierte psychosoziale Behandlung mit pharmakologischer Behandlung verbinden, weil bei effektiver Medikation kombinierte Behandlungen die besten Erfolgschancen haben

8. eine integrierte Behandlung für weitere psychiatrische oder somatische Krankheiten (Komorbidität) bieten

9. berücksichtigen, dass medikamentöser Entzug nur den Behandlungsbeginn darstellt und wenig zur Veränderung der Drogenabhängigkeit beitragen kann; längerfristige Behandlung ist darüber hinaus notwendig

10. das Ziel der Abstinenz bezogen auf alle Drogen, nicht nur auf Kokain, unterstützen

11. berücksichtigen, dass Behandlung nicht freiwillig sein muss, um effektiv zu sein

12. eine Überwachung von möglichem Drogenkonsum durch Urinanalysen und weitere biologische Tests einbeziehen

13. eine Untersuchung auf HIV/AIDS, Hepatitis B und C, TB und andere Infektionserkrankungen sowie eine Beratung zur Prävention und den Umgang mit der Erkrankung anbieten

14. die Teilnahme an Selbsthilfegruppen unterstützen, da diese die professionelle Behandlung ergänzen und ausweiten können

15. last but not least: Die Heilung von Sucht ist häufig ein langfristiger Prozess und kann vielfache Episoden der Behandlung erfordern.

\section{Empirisch gesicherte Behandlungen}

Gegenwärtig gibt es drei bekannte psychotherapeutische Modelle der Verhaltenstherapie für Kokainabhängigkeit, die besonders viel versprechend sind; alle diese sind empirisch gestützt:

- Individual Drug Counseling/Individuelle Drogenberatung

- Cognitive-Behavioral Therapy (Relapse Prevention)/Kognitive Verhaltenstherapie (Rückfallprävention)

- Community Reinforcement Approach (Contingency Management)/Gemeindenahes Verstärkermodell (Contingency Management)

\section{Individuelle Drogenberatung}

Individuelle Drogenberatung (Individual Drug Counseling, IDC) $[9,10]$ ist unmittelbar auf die Reduktion bzw. Abstinzenz von Drogenkonsum ausgerichtet. Sie richtet sich gleichfalls auf die Bereiche, die durch die Abhängigkeit beeinträchtigt sind - wie beispielsweise Erwerbsstatus, Familien- und Sozialbeziehungen, illegale Aktivitäten - sowie auf Inhalt und Struktur des Heilungsprogramms des Patienten. Sie konzentriert sich auf kurzfristige Verhaltensziele, die dem Patienten dabei helfen, sich von der Substanz zu distanzieren und dann Hilfsstrategien zu entwickeln, um abstinent zu bleiben. Als Ergänzung der professionellen Behandlung unterstützt dieses Modell stark die Teilnahme an 12-Schritte- oder anderen Selbsthilfeprogrammen. Wenn nötig, werden Überweisungen $\mathrm{zu}$ anderen Hilfsangeboten wie z.B. medizinische, psychiatrische und Arbeitsberatung vorgenommen.

Die IDC wurde aus den allgemeinen Drogenberatungstechniken für die NIDA-Kokain-Psychotherapiestudie (siehe unten) entwickelt. In dieser, an mehreren Orten durchgeführten Studie an 487 kokainabhängigen Erwachsenen half IDC mit einer zusätzlichen Gruppe(nberatung) den Patienten in größerem Maß, ihren
Drogenkonsum zu reduzieren, als dies bei kognitiver Therapie mit zusätzlicher Gruppe oder bei einem psychodynamischen Ansatz plus Gruppe der Fall war. In dieser Studie dauerte die Behandlung 6 Monate, einschließlich drei anschließender Kontrollsitzungen. In den ersten drei Monaten war die Behandlungshäufigkeit zweimal pro Woche und in den Monaten 4-6 einmal pro Woche. Während der gesamten 6-monatigen Behandlungsdauer wurde einmal wöchentlich zusätzlich Gruppentherapie angeboten.

\section{Kognitive Verhaltenstherapie}

Kognitive Verhaltenstherapie (Cognitive-Behavioral Therapy, CBT) [11] basiert auf Modellen der Rückfallprävention [12] und wurde ursprünglich für die Behandlung von Problemtrinkern entwickelt, später jedoch für Kokainabhängigkeit angepasst. Kognitive Verhaltensstrategien basieren auf der Theorie, dass Lernprozesse eine maßgebliche Rolle bei der Entwicklung von Verhaltensmustern spielen. In dieser Therapie lernen die Klienten, problematisches Verhalten zu identifizieren und zu korrigieren. Rückfallprävention umfasst verschiedene kognitive Verhaltensstrategien, um die Abstinenz zu erleichtern und dem Rückfall vorzubeugen. Der CBT-Behandlungsansatz von Kokainabhängigkeit besteht aus einer Anzahl von Strategien, die die Selbstkontrolle fördern sollen. Spezifische Techniken umfassen die Erforschung der positiven und negativen Konsequenzen des fortgesetzten Konsums, Selbstbeobachtung zur Früherkennung von Suchtdruck und Erkennung von Risikosituationen, Entwicklung von Strategien zur Vermeidung von und zum Umgang mit Risikosituationen und dem Wunsch zu konsumieren. Dieses Modell versucht den Klienten dabei zu helfen, Probleme vorauszusehen und effektive Coping-Strategien zu entwickeln.

CBT gehört zu den am häufigsten evaluierten psychosozialen Ansätzen zur Behandlung von Substanzabhängigkeit. Ein Forschungsüberblick [13] konnte zeigen, dass, verglichen mit nicht behandelten Kontrollgruppen, die Effektivität von CBT bei der Behandlung verschiedener Suchtmittelerkrankungen bewiesen wurde. Die Ergebnisse einiger Studien zeigen, dass CBT im Vergleich zu den anderen häufig angewandten Behandlungen ähnlich effektiv, aber nicht effektiver ist als andere Ansätze, während andere Studien die Überlegenheit von CBT, vor allem bei Patienten mit schwerer Kokainabhängigkeit und schwerer Psychopathologie, nahe legen.

Carroll et al. [14] verglichen für die Behandlung von Kokainabhängigkeit CBT mit der Interpersonellen Psychotherapie (IPT). Beide untersuchten Gruppen reduzierten ihren Kokainkonsum, insgesamt waren die Unterschiede jedoch nicht signifikant. Bei der Untergruppe von Patienten mit schwerem Kokainkonsum zeigte sich, dass die CBT-Behandelten eher abstinent wurden als die IPT-Behandelten (54\% vs. 9\%).

In einer weiteren 4-Felder-Studie wurde CBT vs. Clinical Management (CM) mit Desipramin vs. Plazebo verglichen [11]. Nach 12 Wochen Behandlung zeigten alle vier Gruppen signifikante Verbesserungen bei der Verringerung des Kokainkonsums. Hierbei gab es keine signifikanten Unterschiede bezüglich der Wirkung von Psychotherapie bzw. Medikation. Dennoch ist zu beachten, dass die Patienten mit der schwersten Kokainabhängigkeit bes- 
ser mit CBT als mit CM abschnitten. Ein interessantes Ergebnis war, dass sich bei der Kontrolluntersuchung nach einem Jahr anscheinend eine verzögerte Wirkung von CBT bemerkbar machte. Nach dem Ende der Behandlung haben die Klienten, die mit CBT behandelt worden waren, ihren Kokainkonsum weiter reduziert, während bei den anderen (CM) der Konsum stabil blieb.

\section{Community-Reinforcement-Ansatz}

Das gemeindenahe Verstärkermodell (Community Reinforcement Approach, CRA) beinhaltet eine „Belohnung“ $[15,16]$. Dies ist eine intensive ambulante Therapie, die ebenfalls ursprünglich für Alkoholabhängige entwickelt wurde und für Kokainabhängige modifiziert worden ist. Primäres Ziel ist, Kokainabstinenz so lange aufrechtzuerhalten, dass die Patienten neue Fähigkeiten erwerben können, die ihnen bei der Beibehaltung des abstinenten Verhaltens helfen.

Die Patienten nehmen an ein oder zwei individuellen Beratungssitzungen in der Woche teil. Im Vordergrund steht die Verbesserung der familiären Beziehungen, das Erlernen verschiedener Strategien zur Minimierung des Drogenkonsums, Arbeitsberatung und die Entwicklung neuer Freizeitaktivitäten sowie Bildung sozialer Netzwerke. Die Patienten unterziehen sich 2- bis 3-mal pro Woche einer Unrinkontrolle und erhalten bei negativen Proben als Anreiz eine Belohnung in Form eines Gutscheins. Der Wert dieses Gutscheins steigt mit der Anzahl der aufeinander folgenden drogenfreien Proben. Die Gutscheine können gegen Waren eingetauscht werden, die mit einem gesunden, nüchternen Leben vereinbar sind. Dieser Ansatz fördert das Interesse der Patienten an der Behandlung und hilft ihnen systematisch, Abstinenzphasen zu erleben.

Viele Studien haben diesen Ansatz (CRA) mit einem Standardmodell der Drogenberatung verglichen, das den speziellen Verhaltensanreiz der Belohnung allerdings nicht beinhaltet. CRA wurde in der Behandlung von Kokainabhängigkeit geprüft, wurde kombiniert mit Disulfiram (Antabus) für die Behandlung von Kokain- und Alkoholabhängigkeit und er wurde in der Methadonsubstitution für Kokain- und Heroinkonsumenten genutzt. In jedem Fall war die Behandlung, die auf dem Community-Reinforcement-Ansatz inklusive Gutschein basierte, erfolgreicher als eine alleinige Drogenberatung. Eine Einschränkung, die manchmal bei diesem Ansatz beobachtet wird, ist die Tendenz, wieder in alte Gewohnheiten zurückzufallen, sobald der Anreiz aufhört. Eine weitere Einschränkung für eine breite Anwendung der Methode in den USA liegt darin, dass Drogenprogramme keine ausreichenden Mittel für die Gutscheine zur Verfügung stellen können.

\section{Die NIDA-Kokain-Therapiestudie}

Die NIDA-Kokain-Therapiestudie war eine an mehreren Standorten stattfindende Untersuchung, die die Effektivität von vier ambulant angewendeten psychosozialen Behandlungen für Kokainabhängige prüfte.

\section{Die Behandlungen waren}

- Gruppendrogenberatung allein (GDC),

- individuelle Drogenberatung (IDC) plus GDC,

- kognitive Therapie (CT) plus GDC,
- Supportive-Expressive Therapy (SE - ein psychodynamischer Ansatz) plus GDC.

Die Behandlung bestand aus einer 6 Monate dauernden aktiven Phase und einer 3-monatigen Auffrischphase. Über die gesamten 6 Monate fanden wöchentlich 1,5 Stunden Gruppensitzungen statt. Ziel war es, den Patienten dabei zu helfen, ihren Kokainkonsum zu reduzieren bzw. zu stoppen und ihnen die Teilnahme an einem 12-Schritte-Programm zu ermöglichen sowie eine Schulung zur HIV-Risikominimierung.

Die individuellen Therapiesitzungen für IDC, CT und SE-Therapie dauerten 50 Minuten und wurden zweimal pro Woche in den ersten 12 Wochen und einmal wöchentlich während der Wochen 13-24 durchgeführt. Monatliche Einzelsitzungen wurden während der Auffrischphase (Monate 7-9) durchgeführt. Der Behandlungserfolg wurde nach 6, 9, 12 und 18 Monaten beurteilt. Hauptzielkriterien waren der Drogenkonsum anhand des Composite Scores des Addiction Severity Index (ASI), das ASI-Item „Kokaingebrauch während der letzten 30 Tage“ und Urinkontrollen.

Die Patienten waren zwischen 18 und 60 Jahre alt, hatten als DSM-IV-Hauptdiagnose eine Kokainabhängigkeit mit gegenwärtiger oder beginnender teilweiser Remission und während der letzten 30 Tage öfter als an einem Tag Kokain konsumiert. Von 2197 gescreenten Patienten erfüllten 1771 die Einschlusskriterien und waren zur Rekrutierung vorgesehen, 937 sind erschienen, 870 begannen mit der Orientierungsphase und 487 schlossen die erforderliche Anzahl an Terminen und Untersuchungen ab und wurden randomisiert.

Die randomisierten Patienten waren im Durchschnitt 34 Jahre, lebten allein (70\%), hatten 13 Jahre Schulbildung und waren erwerbstätig (60\%). Die meisten waren männlich $(77 \%)$ und Weiße (58\%), $40 \%$ waren Afro-Amerikaner und $2 \%$ lateinamerikanischer Herkunft. Crackkonsum war die häufigste Form des Konsums (79\%), $19 \%$ konsumierten Kokain nasal und nur $2 \%$ intravenös. Bei Aufnahme wies der durchschnittliche Patient 7 Jahre Kokaingebrauch auf und berichtete über 10 Tage Kokaingebrauch sowie 7 Tage Alkoholgebrauch im letzten Monat. Nach der Randomisierung hielt etwa die Hälfte der Patienten ihre festgesetzten Termine ein.

In dieser Multicenterstudie an 487 erwachsenen Kokainabhängigen zeigten alle Behandlungsgruppen eine signifikante Reduktion des Kokainkonsums. Die IDC mit Gruppe(nberatung) half jedoch den Patienten eher, ihren Drogenkonsum zu reduzieren als die kognitive Therapie plus Gruppe oder die Supportive-Expressive Psychotherapy plus Gruppe. Die individuelle Therapie plus GDC war den zwei anderen Psychotherapien auch bezogen auf die Anzahl der Tage mit Kokainkonsum im letzten Monat überlegen. Eine Überlegenheit der Psychotherapie gegenüber GDC für Patienten mit schwereren psychiatrischen Störungen konnte nicht bestätigt werden. 
Zusammenfassend haben die meisten Studien zur Psychotherapie bei Kokainabhängigkeit ergeben, dass sie mit einer Reduzierung des Substanzkonsums und Verbesserungen in anderen wichtigen Bereichen einhergehen [14,17]. Es scheint jedoch, dass bestimmte Bedingungen erfüllt sein müssen, um die Chancen zu verbessern, Patienten in einer Therapie zu engagieren und positive Ergebnisse zu erzielen. Gewöhnlich braucht der substanzabhängige Patient mehr Struktur und eine größere Häufigkeit von Sitzungen, als die traditionelle Psychotherapie bietet. Häufige, unter Kontrolle durchgeführte Urin- und Atemtests sind wichtige Aspekte dieser Struktur. Jeder dieser Tests auf Suchtmittel unterstützt Ehrlichkeit und hilft dem Patienten, die Verantwortung für sein Verhalten zu übernehmen. Direkte Rückmeldung der positiven oder negativen Urinkontrolle hilft dem Patienten insofern, dass er merkt, dass der Therapeut sich für ihn interessiert und seine Fortschritte überwacht. Angemessene Konfrontation und Analyse der Gründe für den Drogenkonsum sind bei Rückfällen wichtig, unabhängig davon, ob der Rückfall durch Urinkontrolle, Atemtest oder Selbstaussage des Patienten entdeckt worden ist. Positive Rückmeldung bei sauberen Urintestergebnissen ist eine starke Bestätigung für die Beibehaltung von Abstinenz.

Viele Kliniker finden die Einbeziehung von wichtigen Familienmitgliedern für den Behandlungsprozess ebenfalls hilfreich. Die meisten Berater und Therapeuten richten besondere Aufmerksamkeit auf Faktoren innerhalb der Familie, die die Behandlung unterminieren könnten, wie z.B. Sucht bei einem Familienmitglied, indirekte finanzielle Unterstützung der Sucht („Befähigung“) durch die Gabe anderer Güter, wenn das ganze Geld für Drogen ausgegeben wurde, oder auch die Entwicklung von Familienkrisen als Ergebnis einer Besserung des Patienten. Wenn solche Faktoren bestehen, könnte eine Familientherapie ebenfalls notwendig werden. Mindestens eine kontrollierte Studie über strukturierte Familientherapie, die in Kombination mit Drogenberatung für Methadonsubstituierte angewandt wurde, zeigte positive Ergebnisse [18].

Die meisten der verschiedenen Therapiemodelle bieten hilfreiche Strategien an und vielleicht zeigen zukünftige Studien, dass bestimmte Patientencharakteristika mit größeren Verbesserungen in einer bestimmten Psychotherapie, verglichen mit einer anderen, verbunden sind. Andere Studien haben gezeigt, dass Therapeuten- und Patientenqualitäten, einschließlich des Vorhandenseins psychiatrischer Symptome und Störungen, Einfluss auf das Ergebnis haben $[8,6,19]$. Ein sehr positiver Effekt von Psychotherapie, Beratung und anderen Behandlungen bei Substanzmissbrauch ist die Reduktion des HIV-Risikoverhaltens, ein jüngstes Ergebnis, das sich quer durch alle Therapiemodalitäten zeigt. Dies ist extrem wichtig, besonders für einige Gruppen von Drogenkonsumenten und auch in einem allgemeinen Sinn, d.h. für die öffentliche Gesundheit [20].
${ }^{1}$ National Institute on Drug Abuse Research Report. Cocaine Abuse and Addiction. NIH Publ 1999; No 99-4342

${ }^{2}$ National Drug and Alcoholism Treatment Unit Survey DHHS Publ No ADM-89-1626. Rockville: U.S. Department of Health and Human Services 1982

${ }^{3}$ Onken LS, Blaine JD. Psychotherapy and counseling research in drug abuse treatment: questions, problems, and solutions In: Onken LS, Blaine JD (Hrsg) Psychotherapy and Counseling in the Treatment of Drug Abuse. Rockville, MD: U.S. Department of Health and Human Services, NIDA Res Monogr 1990; 104: 1 -5

${ }^{4}$ Khantzian EJ. The self-medication hypothesis of addictive disorders: focus on heroin and cocaine dependence. Am J Psychiatry 1985; 142: $1259-1264$

${ }^{5}$ Washton AM. Cocaine Addiction: Treatment, Recovery, Relapse Prevention New York: WW Norton 1989

${ }^{6}$ Luborsky L, Crits-Christoph P, McLellan AT. Do therapists vary in their effectiveness? Findings from four outcome studies. Am J Orthopsychiatry 1986; 66: $501-512$

${ }^{7}$ Kleinman PH, Woody GE, Todd TC et al. Crack and cocaine abusers in outpatient psychotherapy In: Onken LS, Blaine JD (Hrsg) Psychotherapy and Counseling in the Treatment of Drug Abuse. Rockville, MD: U.S. Department of Health and Human Services, NIDA Res Monogr 1990; 104: $24-34$

${ }^{8}$ Luborsky L, McLellan AT, Woody GE et al. Therapist success and its determinants. Arch Gen Psychiatry 1985; 42: 602 -611

${ }^{9}$ Mercer DE, Woody GE. Addiction Counseling (unpublished manual) 1992

${ }^{10}$ Mercer D, Woody GE. An Individual Counseling Approach to Treat Cocaine Addiction: The Collaborative Cocaine Treatment Study Model Manual 3 in National Institute on Drug Abuse Therapy Manuals for Drug Addiction NIDA 1999

${ }^{11}$ Carroll KM, Rounsaville BJ, Gordon LT et al. Psychotherapy and pharmacotherapy for ambulatory cocaine abusers. Arch Gen Psychiatry 1994; 51: $177-187$

12 Relapse Prevention: Maintenance Strategies in the Treatment of Addictive Behaviors In: Marlatt GA, Gordon J (Hrsg). New York: Guilford 1985

${ }^{13}$ Carroll KM. Relapse prevention as a psychosocial treatment: A review of controlled clinical trials. Experimental \& Clinical Psychopharmacology 1996; 4: 46-54

${ }^{14}$ Carroll KM, Rounsaville BJ, Treece FH. A comparative trial of psychotherapies for ambulatory cocaine abusers: relapse prevention and interpersonal psychotherapy. Am J Drug Alcohol Abuse 1991; 17: $229-247$

${ }^{15}$ Higgins ST, Budney AJ, Bickel WK, Foerg F, Donham R, Badger G. Incentives improve outcome in outpatient behavioral treatment of cocaine dependence. Archives of General Psychiatry 1994; 51: 568-576

${ }^{16}$ Higgins ST, Budney AJ, Bickel WK, Badger GJ et al. Outpatient behavioral treatment for cocaine dependence: One-year outcome. Experimental \& Clinical Psychopharmacology 1995; 3: 205-212

17 Crits-Christoph P, Siqueland L, Blaine JD, Frank A, Luborsky L, Onken LS, Muenz L, Thase ME, Weiss RD, Gastfriend DR, Woody GE, Barber JP, Butler SF, Daley D, Salloum I, Bishop S, Najavits LM, Lis J. Psychosocial treatments for cocaine dependence: results of the National Institute on Drug Abuse Collaborative Cocaine Treatment Study. Archives of General Psychiatry 1999; 57: 493-502

${ }^{18}$ Stanton MD, Todd TC. The Family Therapy of Drug Abuse and Addiction New York: Guilford 1982

${ }^{19}$ McLellan AT, Woody GE, Luborsky L et al. Is the counselor an „active ingredient" in methadone treatment? An examination of treatment success among four counselors. J Nerv Mental Dis 1988; 176: $423-430$

${ }^{20}$ Metzger DS, Navaline H, Woody GE. Drug abuse treatment as AIDS prevention. Public Health Reports 1998; 113 (Suppl. 1): 97-106 